\title{
Imbalanced Th17/Treg in peripheral blood of adult patients with immunoglobulin A vasculitis nephritis
}

\author{
XUE XUE ${ }^{I}$, QIANG LIU 2,3 , WENCHENG XU ${ }^{2,3}$, JUN YUAN ${ }^{1}$, HUIMIN ZHOU ${ }^{4}$, XINRONG ZOU ${ }^{2,3}$, \\ SIPING HAN ${ }^{2,3}$, XIANGYAN MENG ${ }^{l}$, XIAOQIN WANG ${ }^{2,3}$ \\ 'Department of Internal Medicine, The First Clinical College, Hubei University of Traditional Chinese Medicine, Wuhan, Hubei, China \\ ${ }^{2}$ Hubei Provincial Hospital of Traditional Chinese Medicine, Wuhan, Hubei, China \\ ${ }^{3}$ Hubei Province Academy of Traditional Chinese Medicine, Wuhan, Hubei, China \\ ${ }^{4}$ School of Nursing, Hubei University of Traditional Chinese Medicine, Wuhan, Hubei, China
}

\begin{abstract}
Introduction: Adult immunoglobulin A vasculitis nephritis (IgAVN) was observed to be more severe than the disease in children because it tended to result in a poor prognosis. The present study analyzed the Th17/Treg cell axis in peripheral blood of adult IgAVN patients, aiming to provide new immunological viewpoints for the pathogenesis of adult IgAVN.

Material and methods: Th17 cell and Treg cell frequencies in peripheral blood of healthy subjects $(n=13)$ and adult IgAVN patients $(n=12)$ were analyzed by flow cytometry. Foxp 3 mRNA in peripheral blood of healthy subjects and adult IgAVN patients was detected by RT-PCR. Interleukin (IL)-17 and IL-10 in peripheral blood serum of healthy subjects and adult IgAVN patients were examined by ELISA.

Results: The percentages of $\mathrm{CD}^{+}{ }^{+} \mathrm{Th} 17^{+}$cells in peripheral blood of healthy subjects and adult IgAVN patients were $2.65 \pm 1.55 \%$ and $4.37 \pm 1.68 \%$ respectively. The percentages of Treg cells in peripheral blood of healthy subjects and adult IgAVN patients were $6.44 \pm 2.90 \%$ and $3.91 \pm 1.94 \%$ respectively. The ratio of Th17/Treg in adult IgAVN patients was significantly higher than that of healthy subjects $(p=0.0030)$. Meanwhile, the Foxp 3 mRNA expression of adult IgAVN patients was significantly lower than that of healthy subjects. There was a significant difference in the ratio of IL-17/IL-10 between healthy subjects and adult IgAVN patients $(p<0.0001)$. A significant correlation between red blood cell distribution width (RDW) and the ratio of Th17/Treg in adult IgAVN patients was observed in Spearman correlation analysis $(r=0.6970, p=0.0145)$.
\end{abstract}

Conclusions: Imbalanced Th17/Treg contributed to the complex pathogenesis of adult IgAVN.

Key words: adult, immunoglobulin A vasculitis nephritis, red blood cell distribution width, Th17, Treg.

(Cent Eur J Immunol 2021; 46 (2): 191-198)

\section{Introduction}

Immunoglobulin A vasculitis (IgAV), also referred to as Henoch-Schönlein purpura, is an immune-mediated vasculitis associated with immunoglobulin $\mathrm{A}(\operatorname{Ig} \mathrm{A})$ deposition. It is characterized by leukocytoclastic vasculitis accompanied by IgA immune complexes within affected organs [1, 2]. The main clinical symptoms of IgAV include cutaneous purpura, abdominal pain, hemorrhagic gastroenteritis, arthritis and kidney damage [3]. Among them, IgAV nephritis $(\operatorname{IgAVN})$ is potentially the most severe complication, which usually manifests as proteinuria, hematuria, and even renal failure [4]. IgAVN occurs more frequently in children than in adults and it was listed as the most common secondary glomerular disease in children [5].
However, adult IgAVN was usually observed to be more severe than the disease in children because it tended to result in a poor prognosis [6].

Although the complex pathogenesis of IgAVN has not been fully elucidated yet, recent studies revealed that immune abnormalities played a significant role in the pathogenesis of IgAVN [7]. CD4 ${ }^{+} \mathrm{T}$ cells are important cells involved in the immune response. Regulatory T-cells (Tregs) and Th17 cells, which belong to $\mathrm{CD}^{+} \mathrm{T}$ cell subsets, maintain the body's immune balance. Tregs are responsible for the maintenance of self-tolerance, thus inhibiting autoimmunity, whereas pro-inflammatory Th17-cells contribute to the induction and propagation of inflammation [8]. Tregs, marked by Foxp 3, were reported to secrete IL-10 to prevent inflammatory and autoimmune patholo-

Correspondence: Xiaoqin Wang, Hubei Provincial Hospital of Traditional Chinese Medicine, Wuhan, Hubei, 430061, China; Hubei Province Academy of Traditional Chinese Medicine, Wuhan, Hubei, 430074, China, e-mail: wangxiao773@ hotmail.com Submitted: 4.12.2019; Accepted: 12.08.2020 
gies $[9,10]$. Th17 expressed high levels of IL-17A (commonly referred to as IL-17), which is recognized as one of the key effector cytokines of Th17 cells to promote an inflammatory response [8, 11]. An imbalance of the Th17/ Treg cell axis has been observed in a variety of autoimmune nephropathies, such as IgA nephropathy, minimal change nephrotic syndrome and lupus nephritis [12-14]. An imbalance of the Th17/Treg cell axis also contributed to the pathogenesis of IgAV in children [15].

However, there are few studies to report the imbalance of the Th17/Treg cell axis in adult IgAVN. Thus, we examined the Th17/Treg cell axis in peripheral blood of adult patients with IgAVN in the present study. Recently, red blood cell distribution width (RDW) was also reported to be a novel inflammatory marker in several kinds of inflammatory diseases [16-18]. In the present study, we also examined the relationship between the ratio of Th17/ Treg cells and of adult IgAVN patients, aiming to provide some new immunological viewpoints for the pathogenesis of adult IgAVN.

\section{Material and methods}

\section{Reagent}

PerCP Mouse Anti-Human CD4 (Cat\# 347324), FITC Mouse Anti-Human CD25 (Cat\#555431), PE Mouse antiHuman Foxp 3 (Cat\# 560852), PE Mouse anti-Human IL-17A (Cat\# 560486), Permeabilizing Solution 2 (Cat\# 340973), Lysing Solution (10X) (Cat\# 349202), and Protein Transport Inhibitor (Cat\# 51-2092KZ) were purchased from BD Biosciences, USA. Red Blood Cell Lysis Buffer
(Cat\# 420301) was obtained from Biolegend, Inc. TRIzol solution (Cat\# 15596026) was purchased from Ambon, USA. SYBR Green PCR kit (Cat\# KM4101) and cDNA first strand synthesis kit (Cat\# 639505) were provided by KAPA Biosystems, USA and Takara Bio, Japan respectively. DNase I (Cat\# AM2295) was obtained from Fermentas, Lithuania. PCR primers were synthesized by Wuhan Tianyi Huiyuan Biotechnology Co., Ltd. All other reagents were of the highest quality available from commercial vendors.

\section{Subjects}

This study was approved by the Ethics Committee of Hubei Provincial Hospital of Traditional Chinese Medicine and in accordance with the Declaration of Helsinki (No. hbzy2019-c37-01). The study was also registered in the China Clinical Trial Registration Center (No. CHiCTR1900025155). All IgAVN patients (8 males and 4 females with the age of $39.25 \pm 13.13$ ) and healthy subjects (6 males and 7 females with the age of $32.85 \pm 9.45$ ) provided written informed consent before data collection. Healthy subjects were recruited from the Health Assessment Center in our hospital. They had neither a history of immunological disorders nor a history of taking immunosuppressive drugs. The IgAVN diagnosis was made according to Clinical Guidelines-a division of nephrology edited by the Chinese Medical Association in 2011 [19]. No patient had received glucocorticoids or other immunosuppressive drugs for the past 3 months before enrolling in the study. Basic characteristics of healthy subjects and adult IgAVN patients are provided in Tables 1 and 2 respectively.

Table 1. Characteristics and laboratory data of healthy subjects

\begin{tabular}{|c|c|c|c|c|c|c|c|c|c|c|c|c|}
\hline Case & Gender & Age & $\begin{array}{c}\text { WBC } \\
\left(\times 10^{9} / 1\right)\end{array}$ & $\begin{array}{c}\text { NEU } \\
\left(\times 10^{9} / 1\right)\end{array}$ & $\begin{array}{c}\text { LYM } \\
\left(\times 10^{9} / 1\right)\end{array}$ & $\begin{array}{l}\text { MONO } \\
\left(\times 10^{9} / 1\right)\end{array}$ & $\begin{array}{c}\text { PLT } \\
\left(\times \mathbf{1 0}^{9} / \mathrm{l}\right)\end{array}$ & $\begin{array}{c}\text { RDW } \\
(\%)\end{array}$ & $\begin{array}{c}\text { HGB } \\
(\mathrm{g} / \mathrm{l})\end{array}$ & U-PRO & U-BLD & $\begin{array}{c}\text { eGFR } \\
\left(\mathrm{ml} / \mathrm{min} / 1.73 \mathrm{~m}^{2}\right)\end{array}$ \\
\hline 1 & Female & 28 & 4.65 & 3.26 & 1.16 & 0.22 & 126 & 12.6 & 138 & - & - & 115.5 \\
\hline 2 & Female & 34 & 5.09 & 2.89 & 1.89 & 0.26 & 224 & 12.2 & 122 & - & - & 164 \\
\hline 3 & Male & 26 & 6.12 & 3.33 & 2.15 & 0.42 & 204 & 12.8 & 156 & - & - & 116.6 \\
\hline 4 & Male & 28 & 4.98 & 3 & 1.51 & 0.35 & 214 & 14.3 & 144 & - & - & 127 \\
\hline 5 & Female & 26 & 5.18 & 3.25 & 1.57 & 0.28 & 215 & 12.4 & 122 & - & - & 108.5 \\
\hline 6 & Male & 27 & 6.43 & 3.02 & 2.86 & 0.41 & 232 & 12.6 & 148 & - & - & 118.8 \\
\hline 7 & Male & 47 & 5.67 & 3.54 & 1.59 & 0.36 & 277 & 12.8 & 151 & - & - & 112.1 \\
\hline 8 & Female & 26 & 6.96 & 3.48 & 2.94 & 0.39 & 282 & 11.4 & 142 & - & - & 125.3 \\
\hline 9 & Male & 38 & 5.02 & 2.4 & 2.22 & 0.32 & 192 & 11.5 & 157 & - & - & 121.6 \\
\hline 10 & Male & 37 & 6.33 & 3.91 & 1.97 & 0.39 & 328 & 12.9 & 155 & - & - & 106.5 \\
\hline 11 & Female & 27 & 6.39 & 3.41 & 2.42 & 0.42 & 200 & 12.2 & 120 & - & - & 135.9 \\
\hline 12 & Female & 27 & 5.31 & 2.82 & 1.97 & 0.31 & 185 & 12.3 & 123 & - & - & 203.9 \\
\hline 13 & Female & 56 & 6.85 & 3.58 & 2.74 & 0.39 & 125 & 13.2 & 134 & - & - & 95 \\
\hline
\end{tabular}


Table 2. Characteristics and laboratory data of adult IgAVN patients

\begin{tabular}{|c|c|c|c|c|c|c|c|c|c|c|c|c|c|c|c|}
\hline Case & Gender & Age & $\begin{array}{l}\text { Duration } \\
\text { of HSPN } \\
\text { (months) }\end{array}$ & $\begin{array}{c}\text { WBC } \\
\left(\times 10^{\circ} / 1\right)(\end{array}$ & $\begin{array}{l}\text { NEU } \\
\left(\times 10^{9} / l\right)(\end{array}$ & $\begin{array}{c}\text { LYM } \\
\left(\times 10^{9} / /\right)\end{array}$ & $\begin{array}{l}\text { MONO } \\
\left(\times 10^{9} / 1\right)\end{array}$ & $\begin{array}{c}\text { PLT } \\
\left(\times 10^{9} / 1\right)\end{array}$ & $\begin{array}{l}\text { RDW } \\
(\%)\end{array}$ & $\begin{array}{c}\text { HGB } \\
(\mathrm{g} / \mathrm{l})\end{array}$ & $\begin{array}{c}\text { ALB } \\
(\mathrm{g} / \mathrm{l})\end{array}$ & U-PRO & U-BLD & $\begin{array}{c}\text { BLO } \\
(\text { per } \mu \mathrm{l})\end{array}$ & $\begin{array}{c}\text { eGFR } \\
(\mathrm{ml} / \mathrm{min} / \\
\left.1.73 \mathrm{~m}^{2}\right)\end{array}$ \\
\hline 1 & Male & 38 & 18 & 5.28 & 3.27 & 1.46 & 0.35 & 137 & 13.2 & 156 & 44.6 & + & ++ & 161.5 & 144.2 \\
\hline 2 & Female & 28 & 72 & 6.73 & 4.65 & 1.54 & 0.50 & 252 & 13.9 & 134 & 47.9 & + & ++ & 41.5 & 109.2 \\
\hline 3 & Male & 35 & 180 & 6.10 & 3.40 & 1.6 & 0.50 & 192 & 12.9 & 127 & 40.4 & ++ & ++ & 484.8 & 65.0 \\
\hline 4 & Male & 47 & 31 & 6.46 & 3.76 & 1.47 & 0.58 & 222 & 12.5 & 166 & 45.7 & + & \pm & 7.4 & 90.3 \\
\hline 5 & Female & 31 & 31 & 8.46 & 4.88 & 3.16 & 0.58 & 335 & 13.0 & 126 & 43.0 & + & ++ & 518 & 205.3 \\
\hline 6 & Female & 28 & 73 & 7.04 & 4.40 & 2.00 & 0.50 & 330 & 13.5 & 131 & 48.1 & \pm & + & 32.8 & 158.0 \\
\hline 7 & Male & 34 & 69 & 4.70 & 2.60 & 1.70 & 0.30 & 181 & 12.5 & 155 & 46.2 & \pm & \pm & 3.7 & 114.7 \\
\hline 8 & Male & 27 & 32 & 7.40 & 4.60 & 2.20 & 0.50 & 185 & 12.8 & 150 & 45.9 & + & + & 121.9 & 122.9 \\
\hline 9 & Male & 48 & 28 & 9.20 & 5.50 & 3.00 & 0.50 & 207 & 14.3 & 148 & 43.8 & ++ & \pm & 2.4 & 69.7 \\
\hline 10 & Male & 29 & 57 & 5.86 & 2.91 & 2.50 & 0.33 & 346 & 14.7 & 119 & 42.8 & ++ & ++ & 208.1 & 92.9 \\
\hline 11 & Male & 65 & 70 & 5.90 & 3.50 & 1.70 & 0.30 & 236 & 14.1 & 119 & 40.0 & ++ & + & 48.5 & 88.5 \\
\hline 12 & Female & 61 & 25 & 10.65 & 6.49 & 3.39 & 0.55 & 379 & 12.6 & 110 & 38.3 & + & + & 148.9 & 60.8 \\
\hline
\end{tabular}

\section{Analysis of Th17 cells in peripheral blood}

Fresh blood samples were treated according to the instructions of Human Th1/Th2/Th17 Phenotyping Kit (BD Pharmingen, BD Biosciences, USA). After that, cells were fixed and permeabilized, and PerCP Mouse Anti-Human CD4 and PE Mouse anti-Human IL-17A antibodies were added to incubate with cells at room temperature for 30 minutes according to the manufacturer's instructions. Then cells were washed, resuspended and measured on the machine. Isotype controls were used to confirm antibody specificity. Data were analyzed by a FACSCalibur flow cytometer equipped with CellQuest software (Becton Dickinson, USA).

\section{Analysis of Treg cells in peripheral blood}

Cells were incubated with PerCP Mouse Anti-Human CD4 and FITC Mouse Anti-Human CD25 at room temperature for 20 minutes. Then, $2 \mathrm{ml}$ of Lysing Solution (1X) were added, followed by 10 minutes' incubation at room temperature in the dark. Cells continued to be stained with PE Mouse anti-Human Foxp 3 antibody for 20 minutes in the dark. After washing and resuspending, cells were measured on the machine. Data were analyzed by a FACSCalibur flow cytometer equipped with CellQuest software.

\section{Detection of Foxp3 mRNA in peripheral blood}

Frozen blood samples were treated by Red Blood Cell Lysis Buffer. Then, $1.0 \mathrm{ml}$ of TRIzol solution was added to a centrifuge tube of $1 \times 10^{6}$ cells to extract total RNA, and cDNA synthesis was carried out according to the manufac- turer's instructions. The specific sequence of Foxp3 was set up as 5'-ACCAAGGCTTCATCTGTG-3', 5'-CTCTGGGAATGTGCTGTT-3', product size 216 bp. $\beta$-actin was used to as a control, 5'-ACACTGTGCCCATCTACG-3', 5'-TGTCACGCACGATTTCC-3', product size $153 \mathrm{bp}$. Real-time PCR was performed according to the description of the SYBR Green PCR kit. The reaction procedure was set up as follows: $95^{\circ} \mathrm{C}, 3 \mathrm{~min} ; 95^{\circ} \mathrm{C}, 5 \mathrm{~s} ; 56^{\circ} \mathrm{C}$, $10 \mathrm{~s} ; 72^{\circ} \mathrm{C}, 25 \mathrm{~s} ; 39$ cycles; $65^{\circ} \mathrm{C}, 5 \mathrm{~s} ; 95^{\circ} \mathrm{C}, 50 \mathrm{~s}$. After the amplification, the data were analyzed by the Fluorescent Quantitative PCR Amplification Instrument (CFX-Connect 96, Bio-Rad, USA).

\section{Quantification of IL-17 and IL-10 in serum}

Concentrations of IL-17 and IL-10 in the serum were measured by enzyme-linked immunosorbent assay according to the manufacturer's instructions (IL-17 Cat\#HM10198, IL-10 Cat\#HM10203, Bioswamp, China). Data were collected by a microplate reader (352 type, Labsystems Multiskan MS, Finland).

\section{Statistical analysis}

Differences of values between healthy subjects and adult IgAVN were analyzed with the Mann-Whitney test. The relationship between red blood cell distribution width and ratio of Th17/Treg in adult IgAVN patients was analyzed by Spearman correlation analysis. These analyses were performed with GraphPad PRISM 8.0.1 (GraphPad Software Inc., San Diego, CA). In each case, two-sided $p$ values $<0.05$ were considered to be significant. 


\section{Results}

Th17 cell and Treg cell frequencies in peripheral blood of healthy subjects and adult IgAVN patients

As shown in Figure 1B, CD4+ $\mathrm{IL} 17 \mathrm{~A}^{+}$was recognized as Th17 cells. The percentages of $\mathrm{CD} 4^{+} \mathrm{Th} 17^{+}$cells in peripheral blood of healthy subjects and adult IgAVN

A

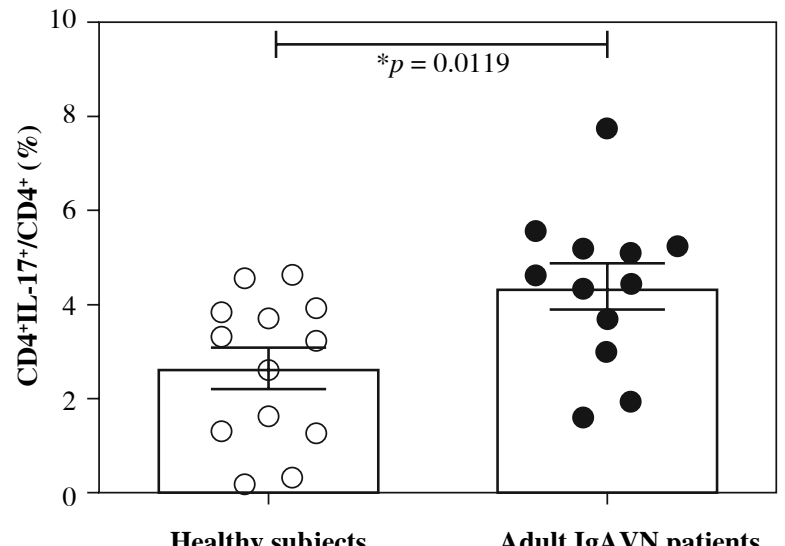

C

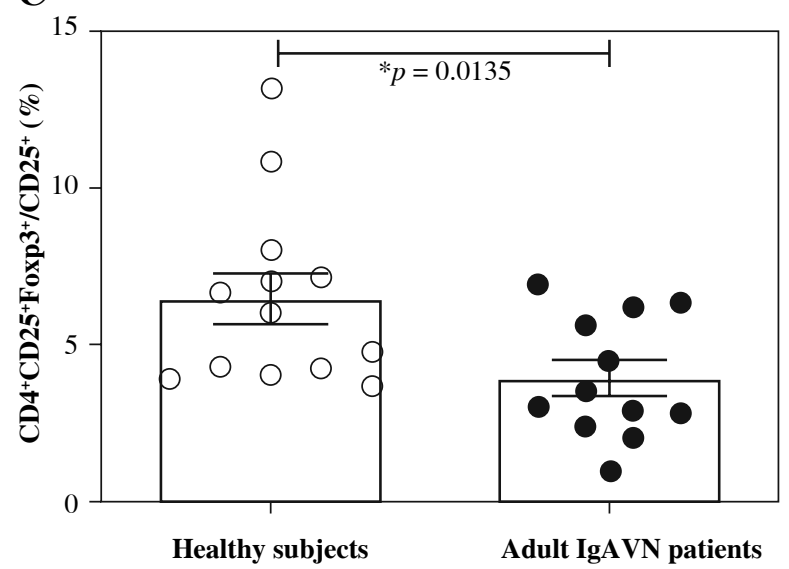

E

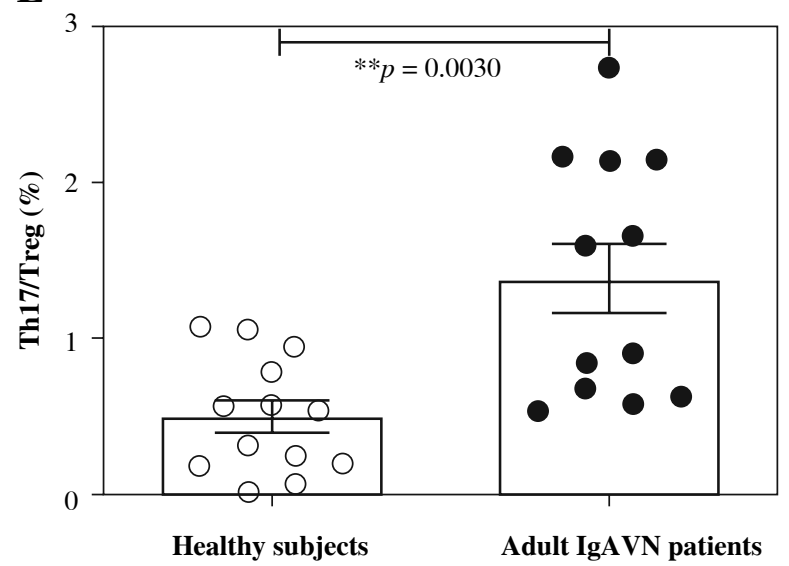

patients were $2.65 \pm 1.55 \%$ and $4.37 \pm 1.68 \%$ respectively (Fig. 1A). The frequency of $\mathrm{CD} 4^{+} \mathrm{Th} 17^{+}$cells of adult IgAVN patients was significantly higher than that of healthy subjects $(p=0.0019)$.

As shown in Figure 1D, CD4 ${ }^{+} \mathrm{CD} 25^{+}$Foxp $3{ }^{+}$was recognized as Treg cells. The percentages of Treg cells in peripheral blood of healthy subjects and adult IgAVN patients were $6.44 \pm 2.90 \%$ and $3.91 \pm 1.94 \%$ respectively

\section{B}
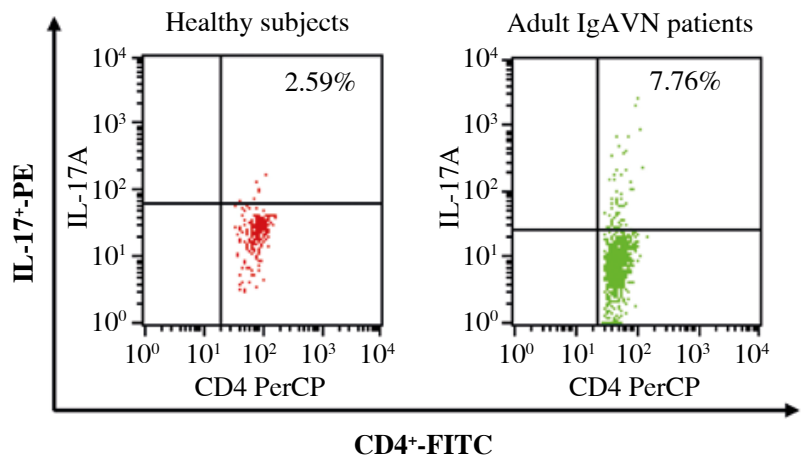

D
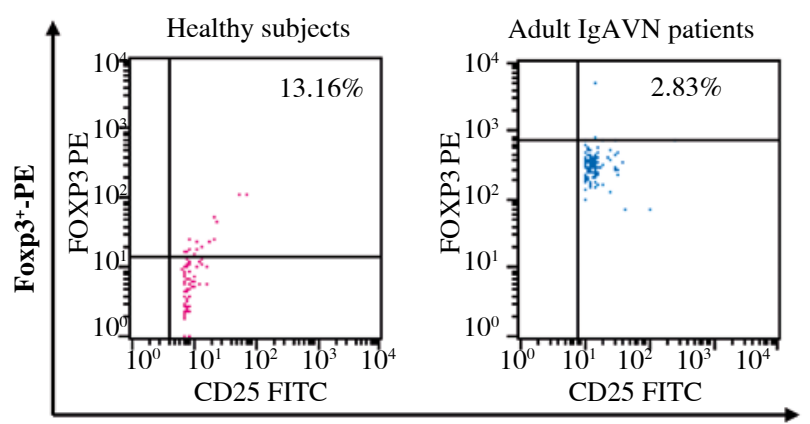

CD25+-FITC $^{+}$

Fig. 1. Th17 cell and Treg cell frequencies in peripheral blood of healthy subjects and adult IgAVN patients. B, D) The typical histograms of Th17 cell and Treg cell frequencies in peripheral blood of healthy subjects and adult IgAVN patients respectively. Statistical results are summarized in A (Th17 cells) and $\mathbf{C}$ (Treg cells). E) The ratio of Th17 and Treg cells in peripheral blood of healthy subjects and adult IgAVN patients 
(Fig. 1C). The frequency of Treg cells of adult IgAVN patients was significantly lower than that of healthy subjects $(p=0.0135)$.

Therefore, an imbalance of Th17/Treg was observed in adult IgAVN patients (Fig. 1E). The ratio of Th17/Treg in adult IgAVN patients was significantly higher than that of healthy subjects $(p=0.0030)$.

\section{Foxp3 mRNA expression in peripheral blood of healthy subjects and adult IgAVN patients}

Next, we continued to examine the Foxp3 mRNA expression in peripheral blood of healthy subjects and adult IgAVN patients. As shown in Figure 2, the Foxp3 mRNA expression of adult IgAVN patients was significantly lower than that of healthy subjects $(p=0.0336)$.

\section{IL-17 and IL-10 in peripheral blood serum of healthy subjects and adult IgAVN patients}

We also detected the IL-17 and IL-10 in peripheral blood serum of healthy subjects and adult IgAVN patients using ELISA. The data are provided in Figure 3. The concentration of IL-17 in peripheral blood serum of adult IgAVN patients $(413.29 \pm 84.30 \mathrm{pg} / \mathrm{ml})$ was significantly higher than that of healthy subjects $(167.09 \pm 24.97 \mathrm{pg} / \mathrm{ml})$ with a $p$ value lower

A

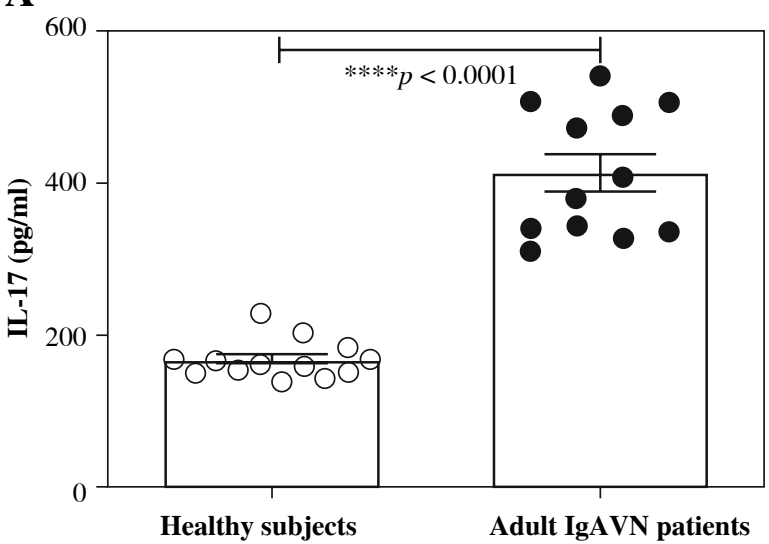

C

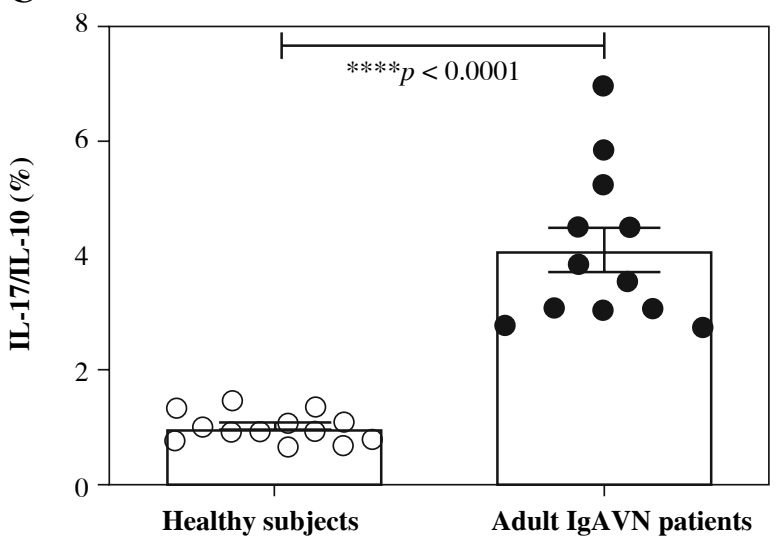

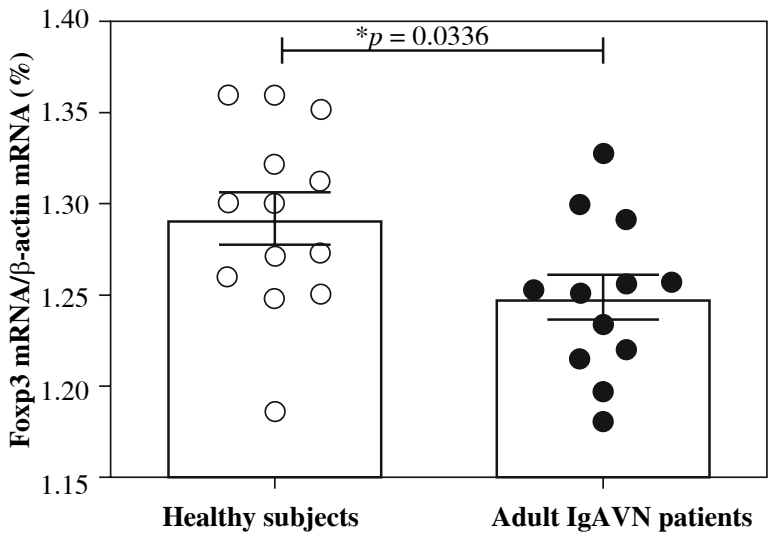

Fig. 2. Expression of Foxp3 mRNA in peripheral blood of healthy subjects and adult IgAVN patients

than 0.0001 (Fig. 3A). However, the concentration of IL-10 in peripheral blood serum of adult IgAVN patients (104.53 $\pm 13.72 \mathrm{pg} / \mathrm{ml}$ ) was significantly lower than that of healthy subjects $(174.21 \pm 32.18 \mathrm{pg} / \mathrm{ml})$ with a $p$ value lower than 0.0001 (Fig. 3B). Thus, there was a significant difference in the ratio of IL-17/IL-10 between healthy subjects and adult IgAVN patients $(p<0.0001$, Fig. 3C).

B

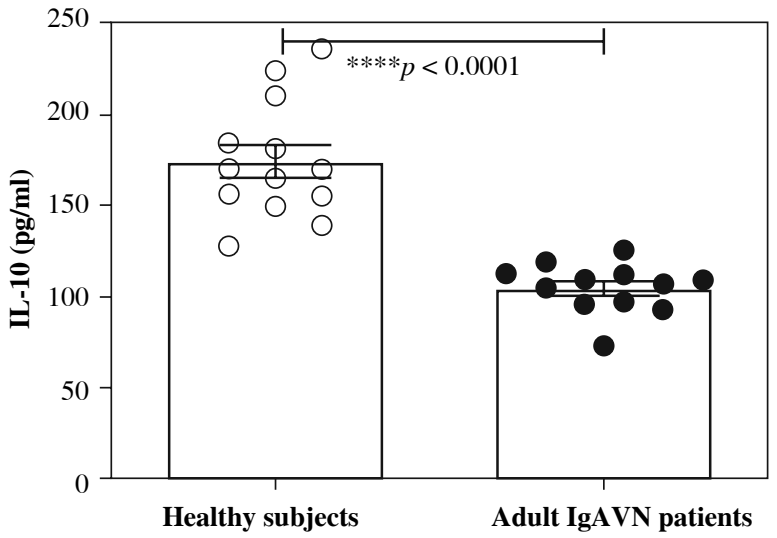

Fig. 3. Concentrations of IL-17 (A) and IL-10 (B) in peripheral blood serum of healthy subjects and adult IgAVN patients. C) The ratio of IL-17/IL-10 
A

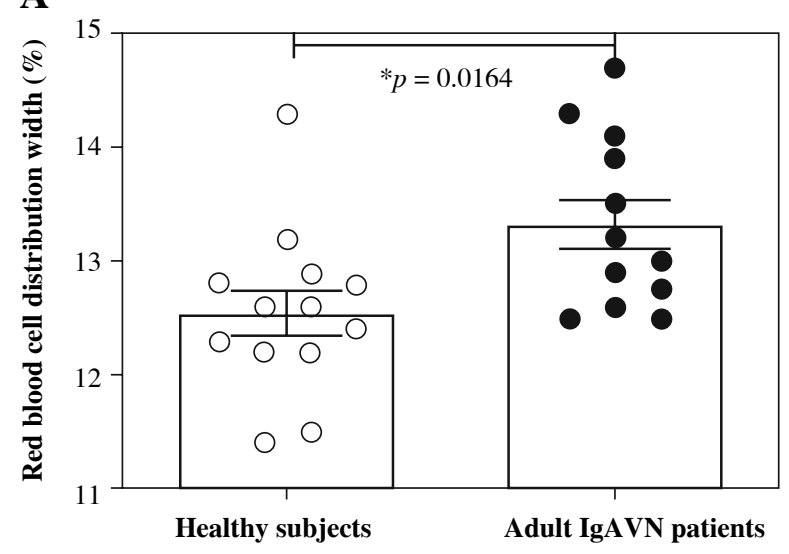

B

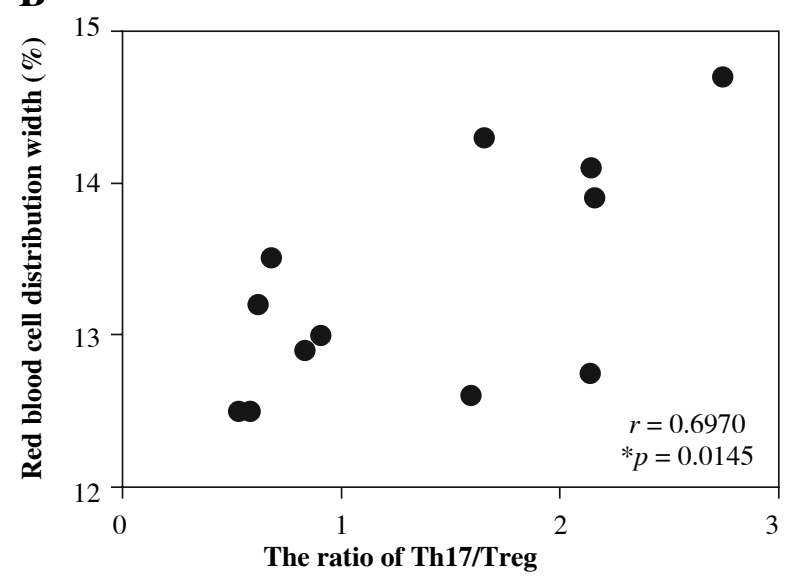

Fig. 4. Relationship between red blood cell distribution width and ratio of Th17/Treg in adult IgAVN patients. A) The significant differences in red blood cell distribution width between healthy subjects and adult IgAVN patients. B) The relationship between red blood cell distribution width and ratio of Th17/Treg in adult IgAVN patients

\section{Relationship between RDW and ratio of Th17/ Treg in adult IgAVN patients}

The significant differences of RDW between healthy subjects and adult IgAVN patients, as shown in Figure 4A, reminded us to examine the relationship between RDW and the ratio of Th17/Treg in adult IgAVN patients. There was a significant correlation between RDW and the ratio of Th17/Treg in adult IgAVN patients in Spearman correlation analysis ( $r=0.6970, p=0.0145$, Fig. 4B).

\section{Discussion}

A national cross-sectional survey in China reported that IgAVN was listed as one of the most common secondary glomerular diseases among children [20]. Most pediatric IgAVN patients present with hematuria and/or low-grade proteinuria, and their prognosis is generally satisfactory [7]. Nearly $5-15 \%$ of pediatric IgAVN patients were reported to progress to chronic renal failure [21]. However, this rate was approximately $30 \%$ in adult IgAVN patients [22]. The present study was designed to investigate the possible immunological pathogenesis of adult IgAVN with a focus on the Th17/Treg cell axis. According to our best knowledge, it is the first report of imbalanced Th17/Treg in peripheral blood of adult IgAVN patients. Moreover, the significant correlation between RDW and the ratio of Th17/Treg in adult IgAVN patients seems to be a new finding.

$\mathrm{CD} 4^{+} \mathrm{Th} 17^{+}$cells and their effector cytokines mediate the crucial crosstalk between the immune system and tissues, and are involved in the pathogenesis of many autoimmune diseases [23]. In Figure 1A, we also observed that the frequency of $\mathrm{CD} 4^{+} \mathrm{Th} 17^{+}$cells of adult IgAVN patients was significantly higher than that of healthy subjects
( $p=0.0019)$. IL-17 was one of the key effector cytokines of Th17 cells $[8,11]$. It was reasonable to obtain a higher concentration of IL-17 in the serum of adult IgAVN patients in Figure 3A $(p<0.0001)$. Treg cells, most of which are produced by the normal thymus as a functionally mature T-cell subpopulation, play key roles in the maintenance of immunologic self-tolerance and negative control of a variety of physiological and pathological immune responses [24]. We also observed that the frequency of Treg cells of adult IgAVN patients was significantly lower than that of healthy subjects ( $p=0.0135$, Fig. 1C). Natural Tregs specifically express Foxp3, a transcription factor that plays a critical role in their development and function [24]. As shown in Figure 2, the Foxp3 mRNA expression of adult IgAVN patients was significantly lower than that of healthy subjects $(p=0.0336)$, which was consistent with the above result. Consequently, as an important mediator of Treg suppression, the concentration of IL-10 in the serum of adult IgAVN patients was significantly lower than that of healthy subjects, as shown in Figure 3B [25]. All these findings strongly suggested that imbalanced Th17/Treg existed in adult IgAVN patients (Figs. 1E and 3C).

Red blood cell distribution width is a simple and inexpensive parameter, which reflects the degree of heterogeneity of erythrocyte volume (conventionally known as anisocytosis), and is traditionally used in laboratory hematology for differential diagnosis of anemias [26]. Recent studies revealed that RDW levels in IgAVN were significantly higher than those in IgAV without nephritis, and RDW would be useful in predicting the presence of crescents on histopathology [27, 28]. Our observation was consistent with these studies (Fig. 4A). RDW was also reported to be a novel inflammatory marker in several kinds of inflammatory diseases, such as septic shock, inflammatory 
bowel disease, and acute appendicitis [16-18]. Distortion of the Th17/Treg balance favoring the pro-inflammatory Th17 side was suspected to contribute to exacerbation of autoimmune disorders [8]. Therefore, we were reminded to examine the correlation of RDW and the ratio of Th17/ Treg in adult IgAVN patients (Fig. 4B). A significant correlation between RDW and the ratio of Th17/Treg in adult IgAVN patients was observed in the present study $(r=0.6970, p=0.0145)$. However, how RDW affects the Th17/Treg cell axis in adult IgAVN patients needs to be addressed in the future.

In the present study, we found imbalanced Th17/Treg in adult IgAVN. This imbalance of the Th17/Treg cell axis was also observed in IgA nephropathy patients [13]. On the other hand, both IgAVN and IgA nephropathy are characterized by circulating immune complexes and their mesangial deposition induced by galactose-deficient IgA1 (Gd-IgA1) [7, 29]. The above information suggests that regulation of the Th17/Treg cell axis might be helpful to reduce the production of circulating immune complexes for both IgAVN and IgA nephropathy.

Numerous studies demonstrated that adult IgAVN patients have more severe renal histopathological changes than pediatric patients $[22,30,31]$. Although Gd-IgA1 was identified to be involved in the pathogenesis of IgAVN, no significant differences were observed between the GdIgA1 level in adult and child IgAVN patients [32, 33]. Until now, the difference in the immunological pathogenesis of adult and child IgAVN is still unclear. The imbalance of the Th17/Treg cell axis plays a significant role in the pathogenesis of a variety of autoimmune nephropathies [12-14]. It is certain that Th17/Treg cell axis imbalance exists in both adult and child IgAVN as reported in the present and previous studies [15]. However, whether the imbalance of the Th17/Treg cell axis is more severe in adult IgAVN than pediatric patients should continue to be investigated in the future.

\section{Acknowledgments}

This study was supported by the QingMiao Plan of Hubei University of Traditional Chinese Medicine (2019ZZX007), Japan China Sasakawa Medical Fellowship (2017816), Collaborative Innovation Research Fund of Institute of Basic Theory of Chinese Medicine, China Academy of Chinese Medical Sciences and Hubei Provincial Hospital of Traditional Chinese Medicine (YZ-1848), National Natural Science Foundation of China (81874439) and Research Project for Practice Development of National Traditional Chinese Medicine Clinical Research Bases (JDZX2015194).

The authors declare no conflict of interest.

\section{References}

1. Zou XR, Xue X, Wang CJ, et al. (2018): A Chinese cohort study on Henoch-Schönlein purpura nephritis: design and methods. Eur J Inflamm 16: 2058739218778936.

2. Heineke MH, Ballering AV, Jamin A, et al. (2017): New insights in the pathogenesis of immunoglobulin A vasculitis (Henoch-Schönlein purpura). Autoimmun Rev 16: 1246-53.

3. Cattran DC, Feehally J, Cook HT, et al. (2012): Kidney disease: improving global outcomes (KDIGO) glomerulonephritis work group. KDIGO clinical practice guideline for glomerulonephritis. Kidney Int Suppl. 2: 139-274.

4. Nikibakhsh AA, Mahmoodzadeh H, Karamyyar M, et al. (2010): Treatment of complicated henoch-schonlein purpura with mycophenolate mofetil: a retrospective case series report. Int J Rheumatol 2010: 254316.

5. Yin XL, Zou MS, Zhang Y, et al. (2013): Twenty-three-year review of disease patterns from renal biopsies: an experience from a pediatric renal center. J Nephrol 26: 699-707.

6. Shah R, Ramakrishnan M, Vollmar A, et al. (2017): Henoch-Schonlein purpura presenting as severe gastrointestinal and renal involvement with mixed outcomes in an adult patient. Cureus 9: e1088.

7. Kawasaki Y (2011): The pathogenesis and treatment of pediatric Henoch-Schonlein purpura nephritis. Clin Exp Nephrol 15: 648-657.

8. Fasching P, Stradner M, Graninger W, et al. (2017): Therapeutic potential of targeting the Th17/Treg axis in autoimmune disorders. Molecules 22: 134.

9. Saraiva M, O'Garra A (2010): The regulation of IL-10 production by immune cells. Nat Rev Immunol 10: 170-181.

10. Hori S, Nomura T, Sakaguchi S (2003): Control of regulatory $\mathrm{T}$ cell development by the transcription factor Foxp3. Science 299: 1057-1061.

11. Annunziato F, Cosmi L, Liotta F, et al. (2012): Defining the human T helper 17 cell phenotype. Trends Immunol 33: 505-512.

12. Liu LL, Qin Y, Cai JF, et al. (2011): Th17/Treg imbalance in adult patients with minimal change nephrotic syndrome. Clin Immunol 139: 314-320.

13. Ruszkowski J, Lisowska KA, Pindel M, et al. (2019): T cells in IgA nephropathy: role in pathogenesis, clinical significance and potential therapeutic target. Clin Exp Nephrol 23: 291-303.

14. Jakiela B, Kosalka J, Plutecka H, et al. (2018): Facilitated expansion of Th17 cells in lupus nephritis patients. Clin Exp Immunol 194: 283-294.

15. Chen O, Zhu XB, Ren H, et al. (2013): The imbalance of Th17/Treg in Chinese children with Henoch-Schonlein purpura. Int Immunopharmacol 16: 67-71.

16. Song CS, Park DI, Yoon MY, et al. (2012): Association between red cell distribution width and disease activity in patients with inflammatory bowel disease. Dig Dis Sci 57: 1033-1038.

17. Kim CH, Park JT, Kim EJ, et al. (2013): An increase in red blood cell distribution width from baseline predicts mortality in patients with severe sepsis or septic shock. Crit Care 17: R282.

18. Bozlu G, Taskinlar H, Unal S, et al. (2016): Diagnostic value of red blood cell distribution width in pediatric acute appendicitis. Pediatr Int 58: 202-205.

19. Chinese Medical Association. (2011): Clinical Guidelines a division of nephrology. People's Medical Publishing House, Beijing, China; 67-69.

20. Nie S, He W, Huang T, et al. (2018): The spectrum of biopsy-proven glomerular diseases among children in China: 
a national, cross-sectional survey. Clin J Am Soc Nephrol 13: 1047-1054.

21. Scharer K, Krmar R, Querfeld U, et al. (1999): Clinical outcome of Schonlein-Henoch purpura nephritis in children. Pediatr Nephrol 13: 816-823.

22. Lu S, Liu D, Xiao J, et al. (2015): Comparison between adults and children with Henoch-Schonlein purpura nephritis. Pediatr Nephrol 30: 791-796.

23. Ouyang W, Kolls JK, Zheng Y (2008): The biological functions of $\mathrm{T}$ helper 17 cell effector cytokines in inflammation. Immunity 28: 454-467.

24. Sakaguchi S, Ono M, Setoguchi R, et al. (2006): Foxp3+ $\mathrm{CD} 25+\mathrm{CD} 4+$ natural regulatory $\mathrm{T}$ cells in dominant self-tolerance and autoimmune disease. Immunol Rev 212: 8-27.

25. Hsu P, Santner-Nanan B, Hu M, et al. (2015): IL-10 potentiates differentiation of human induced regulatory $\mathrm{T}$ cells via STAT3 and Foxo1. J Immunol 195: 3665-3674.

26. Salvagno GL, Sanchis-Gomar F, Picanza A, et al. (2015): Red blood cell distribution width: A simple parameter with multiple clinical applications. Crit Rev Clin Lab Sci 52: 86-105.

27. Xu H, Li W, Mao JH, et al. (2017): Association between red blood cell distribution width and Henoch-Schonlein purpura nephritis. Medicine (Baltimore) 96: e7091.

28. Zhu X, Zhang M, Lan F, et al. (2018): The relationship between red cell distribution width and the risk of Henoch-Schonlein purpura nephritis. Br J Biomed Sci 75: 30-35.

29. Davin JC, Ten Berge IJ, Weening JJ (2001): What is the difference between IgA nephropathy and Henoch-Schonlein purpura nephritis? Kidney Int 59: 823-834.

30. Liu Z, Wei YD, Hou Y, et al. (2016): Differences in pathological characteristics and laboratory indicators in adult and pediatric patients with Henoch-Schonlein purpura nephritis. J Huazhong Univ Sci Technolog Med Sci 36: 659-666.

31. 30. Kang Y, Park JS, Ha YJ, et al. (2014): Differences in clinical manifestations and outcomes between adult and child patients with Henoch-Schonlein purpura. J Korean Med Sci 29: 198-203.

32. Zhang L, Han C, Sun C, et al. (2013): Serum levels of alpha-smooth muscle actin and c-Met as biomarkers of the degree of severity of Henoch-Schonlein purpura nephritis. Transl Res 161: 26-36.

33. Sugiyama M, Wada Y, Kanazawa N, et al. (2020): A cross-sectional analysis of clinicopathologic similarities and differences between Henoch-Schönlein purpura nephritis and IgA nephropathy. PLoS One 15: e0232194. 